\title{
BMJ Open Identifying risk factors for progression to critical care admission and death among individuals with acute pancreatitis: a record linkage analysis of Scottish healthcare databases
}

\author{
Damian J Mole, ${ }^{1,2}$ Usha Gungabissoon, ${ }^{3}$ Philip Johnston, ${ }^{4}$ Lynda Cochrane, ${ }^{5}$ \\ Leanne Hopkins, ${ }^{4}$ Grant M A Wyper, ${ }^{4}$ Christos Skouras, ${ }^{2}$ Chris Dibben, ${ }^{6}$ \\ Frank Sullivan, ${ }^{7}$ Andrew Morris, ${ }^{6}$ Hester J T Ward, ${ }^{4}$ Andrew M Lawton, ${ }^{3}$ \\ Peter T Donnan ${ }^{8}$
}

To cite: Mole DJ,

Gungabissoon U, Johnston P, et al. Identifying risk factors for progression to critical care admission and death among individuals with acute pancreatitis: a record linkage analysis of Scottish

healthcare databases. BMJ Open 2016;6:e011474. doi:10.1136/bmjopen-2016011474

- Prepublication history for this paper is available online. To view these files please visit the journal online (http://dx.doi.org/10.1136/ bmjopen-2016-011474).

Received 11 February 2016 Revised 12 May 2016 Accepted 26 May 2016

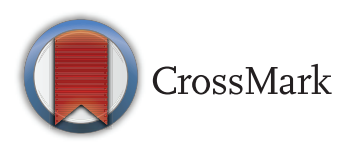

For numbered affiliations see end of article.

Correspondence to Damian J Mole; damian.mole@ed.ac.uk

\section{ABSTRACT}

Objectives: Acute pancreatitis (AP) can initiate systemic complications that require support in critical care (CC). Our objective was to use the unified national health record to define the epidemiology of AP in Scotland, with a specific focus on deterministic and prognostic factors for $C C$ admission in AP.

Setting: Health boards in Scotland $(n=4)$.

Participants: We included all individuals in a retrospective observational cohort with at least one episode of AP (ICD10 code K85) occurring in Scotland from 1 April 2009 to 31 March 2012. 3340 individuals were coded as AP.

Methods: Data from 16 sources, spanning general practice, community prescribing, Accident and Emergency attendances, hospital in-patient, CC and mortality registries, were linked by a unique patient identifier in a national safe haven. Logistic regression and gamma models were used to define independent predictive factors for severe AP (SAP) requiring CC admission or leading to death.

Results: 2053 individuals $(61.5 \%(95 \% \mathrm{Cl} 59.8 \%$ to 63.2\%)) met the definition for true AP (tAP). 368 patients (17.9\% of tAP ( $95 \% \mathrm{Cl} 16.2 \%$ to $19.6 \%)$ ) were admitted to CC. Predictors of SAP were preexisting angina or hypertension, hypocalcaemia and age 30-39 years, if type 2 diabetes mellitus was present. The risk of SAP was lower in patients with multiple previous episodes of AP. In-hospital mortality in tAP was $5.0 \%(95 \% \mathrm{Cl} 4.1 \%$ to $5.9 \%)$ overall and $21.7 \%(95 \% \mathrm{Cl} 19.9 \%$ to $23.5 \%)$ in those with tAP necessitating CC admission.

Conclusions: National record-linkage analysis of routinely collected data constitutes a powerful resource to model CC admission and prognosticate death during AP. Mortality in patients with AP who require CC admission remains high.

\section{Strengths and limitations of this study}

- National record-linkage analysis of routinely collected data constitutes a powerful resource to model critical care (CC) admission and prognosticate death during critical illness.

- This project brought together 16 electronic data sources through a new record-linkage model for Scotland and was at the forefront of the development of the National Data Safe Haven in Scotland.

- For individuals with AP who are not severely ill, electronically formatted routinely recorded clinical data are less comprehensive and this may have led to a negative reporting bias for events not sufficiently severe to need CC admission.

- Although we specifically conducted a data accuracy pilot, comparing electronic records with hand-written notes in four separate study sites, certain variables are likely to be more accurate, for example automatically retrieved Wardwatcher critical care data, than others for example comorbidity and hospital immediate discharge summaries.

- Although specific risk factors can be identified that increase the risk of developing multiple organ dysfunction in AP, it is not pragmatic or straightforward to predict which individual patients are likely to require CC based on their routinely collected medical data.

\section{INTRODUCTION}

Background/rationale

Acute pancreatitis (AP) is an inflammatory disease of the pancreas with potentially devastating consequences for those who suffer a severe 
attack. Individual estimates of the incidence of AP range from 10 to 78 per 100000 per year. ${ }^{1-6}$ AP was found to be the most common reason for hospitalisation with a gastrointestinal disorder in the USA in 2012. ${ }^{7}$ The estimated financial cost for AP-related care in the USA in 2004 was $\$ 3.7$ billion, with AP resulting in 277000 hospital admissions and 475000 ambulatory visits. ${ }^{8}$ The financial cost of treating AP in the UK has been estimated at $£ 200$ million per year. ${ }^{9}$

In $\sim 20 \%$ of patients with AP, severe complications can develop, which may evolve into multiple organ dysfunction syndrome (MODS) requiring critical care (CC) support in high dependency (HDU) or intensive treatment units (ITU). ${ }^{10} 11$ AP-MODS usually occurs rapidly, and features of AP-MODS may be present at the time of presentation to hospital. ${ }^{12}$ Between 2008 and 2010, 5\% of all available intensive care beds in Scotland were taken up by patients with $\mathrm{AP} ;{ }^{9}$ in tertiary referral centres, ITU bed occupancy may reach $9 \% .^{13}$ The epidemiological risk factors for developing organ dysfunction among individuals with AP are not known at present. This knowledge will be important and useful to identify high-risk patients by enhancing the stratification of disease severity, and to influence treatment decisions, plan healthcare, allocate resources and inform trial design. Therefore, there exists a need to understand and identify risk factors for progression to AP-MODS based on laboratory and clinical information leading up to, and at the time of acute presentation to hospital.

The Farr Institute ${ }^{14}$ was conceived with the goal of creating a unified healthcare informatics infrastructure to allow interested parties from government, academia, industry, healthcare providers and other related organisations use linked electronic healthcare data on a national scale in a secure, confidential and streamlined way for medical research within the UK. Given the substantial healthcare burden of AP, the need for improved understanding and treatment of AP and the considerable interest in the development of novel therapies for AP by us ${ }^{15}$ and others, the aim of this study was to harness the power of the Farr Institute capabilities to elucidate the epidemiology of AP with a focus on defining the risk factors for the development of organ failure in AP.

\section{Objectives}

Our specific objectives were to use the Farr Institute infrastructure to:

1. characterise the epidemiology of AP in Scotland,

2. define the determinants of admission to CC and/or death in AP and

3. describe the morbidity, length of CC stay, organ support and mortality of patients with AP admitted to CC.

\section{METHODS}

Study design

We undertook a retrospective observational cohort study of linked healthcare records from 16 electronic data sources in Scotland.

\section{Project structure and study management}

The collaborative core project team consisted of a project manager $(\mathrm{PJ})$, clinician scientists with expertise in AP (DJM and CS), an epidemiologist and statistician from industry (UG and AL), national healthcare data expertise (CD, ADM, FS), data managers, clinical information governance (HW) and clinical statistics and epidemiology expertise (LC and PTD). A project board (including lay membership) met quarterly to oversee the project. The study protocol and analysis plan were agreed in advance by the project board.

\section{Ethical approval, patient confidentiality and data security}

We obtained approvals from: National Services Scotland, Privacy Advisory Committee; National Research Ethics Service; National Research and Development Office; the local Research and Development Office for each Health Board; NHS Board Caldicott Guardians, via the Caldicott Guardians' National Scrutiny Process; Scottish Intensive Care Society Audit Group (SICSAG) Steering Group and General Practitioners (as Caldicott Guardians). The study adhered to strict information governance and security protocols. All primary data were held within the NHS Scotland Information Services Division (ISD) National Safe Haven with access restricted to two named statisticians from the University of Dundee (LC and PTD). Industry partners did not have sight of patient-level data at any stage.

\section{Setting and data sources}

The study cohort consisted of all patients who had a general or acute inpatient or day case record of AP in any coding field, coded as K85 under ICD10 K85 (including K85.0 Idiopathic acute pancreatitis; K85.1 Biliary acute pancreatitis; K85.2 Alcohol-induced acute pancreatitis; K85.3 Drug-induced acute pancreatitis; K85.8 Other acute pancreatitis; K85.9 acute pancreatitis, unspecified), occurring in any of four NHS Health Board areas in Scotland between 1 April 2009 and 31 March 2012. The first episode for any one individual occurring in that time period was taken as the index episode. The combined total population of the four Health Boards was 2063511 (48.9\% of the population of Scotland aged 18 years and over) at the midpoint of the study, and included large conurbations and rural areas. For each patient, data were collected from Accident and Emergency attendances (A\&E2 database), Scottish Morbidity Record 00 (SMR00) Outpatient attendances, SMR01 (General/Acute inpatient and day cases), SMR04 (Mental Health inpatient and day cases), SMR99 (Death registrations), Prescribing Information System (primary care prescribing and dispensing), SICSAG (CC), SCI Stores (laboratory test results) and general practice data. Data were linked within the National Safe Haven by an approved indexing process based on a unique patient identifier (the CHI number). There were no exclusion criteria. A pilot study was performed to assess the coding accuracy of AP in SMR01. 


\section{Definitions and categories}

AP was defined as occurring when any elective or emergency admission was coded as ICD10 K85 in any diagnosis field. To account for inaccuracies of coding, true AP (tAP) was predefined as a subset of AP as: upper abdominal pain or nausea or vomiting at presentation in the clinical records and a serum amylase or lipase result greater than three times the upper limit of the reference range for that laboratory; or CT evidence of AP alone. We predefined severe AP ( $\mathrm{sAP}$ ) as a subset of tAP as: tAP requiring admission to $\mathrm{CC}$ as a part of the same episode or tAP resulting in death during that hospital episode. CC was defined as Level 2, Level 3 or both inpatient care, also known as HDU and ITU, respectively. Aetiology of AP was defined at two levels: (1) in the entire cohort, AP was attributed to gallstones if this (or a related diagnosis, eg, cholecystectomy) was recorded in any coding position; the remaining was regarded as non-gallstone AP; (2) aetiology recorded explicitly in hospital or GP records as gallstones, alcohol or other less common causes. A GP visit was defined as a patient-practice interaction recorded as a visit event in GP records, without being able to differentiate between an actual medical consultation and other, for example obtaining results, visiting the practice nurse or getting a repeat prescription. The day of hospital admission was defined as day 0 , and the next day after hospital admission was defined as day 1 and so on. Overall hospital mortality was defined as death during a single episode of AP occurring prior to hospital discharge. Overall 90-day mortality was defined as any death occurring within 90 days of the first day of an episode of AP.

\section{Variables}

The primary outcome of interest was the development of sAP (admission to CC, tAP death). Secondary outcomes of interest were length of hospital stay in days, length of CG stay in hours and days, maximum number of organs supported, renal replacement therapy, ventilator dependence, duration of enteral nutrition, hospital acquired infection and death among those with sAP admitted to CG. Potential risk factors included patient demographics, measures of socioeconomic deprivation (using the Scottish Index of Multiple Deprivation, ${ }^{16}$ ) aetiology of AP, laboratory and clinical data at the time of presentation to hospital, history of medication use (prior to AP presentation) and clinical/disease history up to the time of AP presentation, the latter two being obtained from patients primary care records in the 12-month period prior to the index episode. Calcium values were corrected for serum albumin concentration.

\section{Record linkage}

Data were linked using established probability and deterministic matching techniques ${ }^{17}$ based on the Howard Newcombe principles. ${ }^{18}$ Linkage was undertaken by a trusted third party. A population spine, which contained the personal identifiers of all individuals who have been in contact with NHS Scotland, was used as an intermediary linkage tool. The process outlined was based on the principles of data linkage outlined in the Scottish Informatics Programme (SHIP) blueprint. ${ }^{19}$ The steps of the process were: the indexing team was supplied the CHI number of each of the cohort members by a single data provider, for which they generated a different record ID number across the range of data sets. Each data provider received a file back with a list of patient CHI numbers and unique person index ID numbers specific to that data set, generated by the indexing team. The data provider then attached the received index ID number to the remaining content of the data set to be provided for linkage and sent this to the research coordinator. The research coordinator sent the file to the linkage agent, which is an automated computer programme. The linkage agent received two files-all the data sets including unique person ID numbers, plus a master control file containing a master person ID and unique person index ID numbers for the entire data set. The linkage agent replaced all the data sets unique person ID numbers with the master person ID number on each of the content data files. The person analysing the data had access to all the records belonging to an individual across all the data sets without sight of personal identifiers.

\section{Statistical methods}

Descriptive analyses were performed according to standard principles using SAS V.9.3 and following the STROBE criteria. ${ }^{20}$ Categorical variables were reported as counts and percentages of the number of valid cases. Continuous variables were described by either mean and SD (for plausibly Normal data) or medians and quartiles (non-Normal data). Between-group comparisons for univariate analyses were assessed by independent samples $t$ test or Mann-Whitney U-test for Normal data and non-Normal data, respectively. Differences in proportions were tested by the $\chi^{2}$ test. To correct for extreme outliers in an otherwise Normally distributed data set, the $5 \%$ trimmed mean was used where stated; this value is the arithmetic mean of data between the 5th and 95th centile. Time-to-event variables were summarised using medians and quartiles. Factors predicting the development of sAP in the tAP cohort were modelled by logistic regression with SAP as a binary dependent variable. Variables were selected on the basis of known clinical importance as well as backward elimination to identify variables significant at the $5 \%$ level. The final model was also assessed for goodness of fit using Akaike's Information Criterion (AIC). Interactions between variables were entered and assessed by adding interaction terms in the model. Predictive performance of the model was assessed by estimation of the area under the receiver operating characteristic curve (AUROC) and calibration assessed using the Hosmer-Lemeshow test. The final model for prediction was based on the largest AUROC. A number of parametric survival models were 
applied to identify factors that predict death during the CC admission. AIC was used to decide on which model was optimal. Model selection criteria were the same as for the logistic regression model.

\section{Imputation of missing data}

For the logistic regression model, where clinically important variables had less than $50 \%$ missing values, multiple imputation was used to ensure that regression analysis was robust, assuming that data were missing at random. In practice, $19 \%$ of calcium measures at index admission were imputed along with $35 \%$ of the primary care flag for alcohol dependency. All other variables in the models were $100 \%$ complete.

\section{RESULTS}

\section{Participants and univariate analysis}

A total of 3340 individuals were identified as having an AP-coded hospital admission, of which 2053 (61.5\%) met the case definition of tAP and were included in the study. Figure 1 shows patient flow in the study. The incidence of an AP-coded hospital admission was 54.0 (95\% CI 41.1 to 70.9) per 100000 per year; the incidence of tAP was 33.1 (95\% CI 24.4 to 46.9) per 100000 per year and the incidence of sAP requiring $\mathrm{CC}$ admission was $5.9 \quad(95 \%$ CI 3.4 to 15.3$)$ per 100000 per year. Demographic characteristics of the cohort are summarised in table 1. Gender distribution was approximately equal across all categories and only increased marginally towards a male preponderance for those with sAP admitted to CC. The mean age of those with sAP was significantly higher than those who did not develop severe disease. Gallstones were co-recorded in $43.8 \%$ of the entire cohort coded as AP. The proportion of patients with gallstone aetiology AP was higher for females. The body mass index (BMI) of patients admitted to CC was on average $1.1 \mathrm{~kg} / \mathrm{m}^{2}$ higher than the remainder of the tAP cohort. A disproportionate number of tAP admissions (35.3\%) were from the most socially deprived quintile; this disproportion was similarly observed in the subset of patients with sAP $(35.0 \%)$. The proportion of patients with tAP who develop sAP and the proportion of those requiring CC admission were not affected by social deprivation (table 1).

\section{Healthcare in general practice prior to study episode of AP}

The GP consultation rate in the year preceding the index AP episode was higher in the subset of patients who died without being admitted to CG than those requiring $\mathrm{CC}$ or those who did not develop sAP (table 2). 25.6\% of patients with an episode of tAP had a previous episode of AP recorded in their GP-held record, but a smaller proportion $(18.5 \%)$ of those with sAP had a previous AP episode documented. Although the aetiology of AP could be attributed to gallstones in $43 \%$ of patients, gallstones were only known to be present (in advance of the index episode) in $17.7 \%$ of those with tAP. A similar proportion (16.5\%) had been admitted to hospital following alcohol misuse prior to the index episode. The proportion with a prior episode of alcohol-related hospital admission was not higher in the sAP group.

There was a greater baseline comorbidity burden among patients with sAP compared to the remainder of the cohort. Pre-existing ischaemic heart disease (previous myocardial infarction (MI) or ischaemic heart disease (IHD)/angina) was more prevalent in those with sAP needing CC admission than those in the tAP cohort as a whole (table 2). Pre-existing type 2 diabetes mellitus was significantly more prevalent in those with sAP requiring CC compared to the tAP cohort. Similarly, chronic obstructive pulmonary disease, hypertension and renal failure (acute and chronic) were significantly more prevalent among the sAP cohort requiring $\mathrm{CC}$ admission (table 2).

The following classes of drugs were prescribed more frequently in those with sAP compared to those not meeting the definition of sAP (nsAP): antidepressants, calcium-altering drugs, neuroleptic agents, oral corticosteroids and statins. The proportions of patients taking each class of drug are presented in table 3.

Endoscopic retrograde cholangiopancreatography had been undertaken in the preceding year in $5.6 \%$ of those admitted with tAP. This proportion was not higher in those with sAP. Coronary stenting had been performed in the preceding year more frequently in those with sAP requiring CC $(3.0 \%)$ compared to the nsAP cohort (1.0\%; table 3$)$.

\section{CC admission}

Of 2053 patients, $368(17.9 \%)$ with tAP were admitted to CC with a median of 1 day (IQR 0,2 ) after admission to hospital (table 4). The 5\% trimmed mean for this time interval was 1.8 days. $27.4 \%$ of patients had two or more $\mathrm{CC}$ admissions in relation to one episode of AP and $6.2 \%$ had three or more CG admissions. Median (IQR) duration of stay for the first CC admission was 68 $(27,139)$ hours. The duration of CC stay was slightly longer when averaged over all CC admissions (median 72 hours, IQR $(29,156)$ ), and when total (sum) CC stay was calculated for any one individual AP episode (ie, episodes during which there was more than one CG stay), the median (IQR) duration was $107(45,221)$ hours. An individual patient with AP requiring $\mathrm{CC}$ admission used $6(3,11)$ days of CC care (table 5$)$.

The median (IQR) APACHE II score on admission to CC was 19 (14, 24). The number of organ systems requiring support is presented in table 4 . A small proportion of patients with sAP $(24 / 368(6.5 \%))$ were admitted for close observation without any recorded organ support. During the initial admission, cardiovascular support with vasopressors was used in $60.3 \%$ of patients with sAP, but this value rose to $65.5 \%$ when all CG admissions were considered. $13.9 \%$ required 


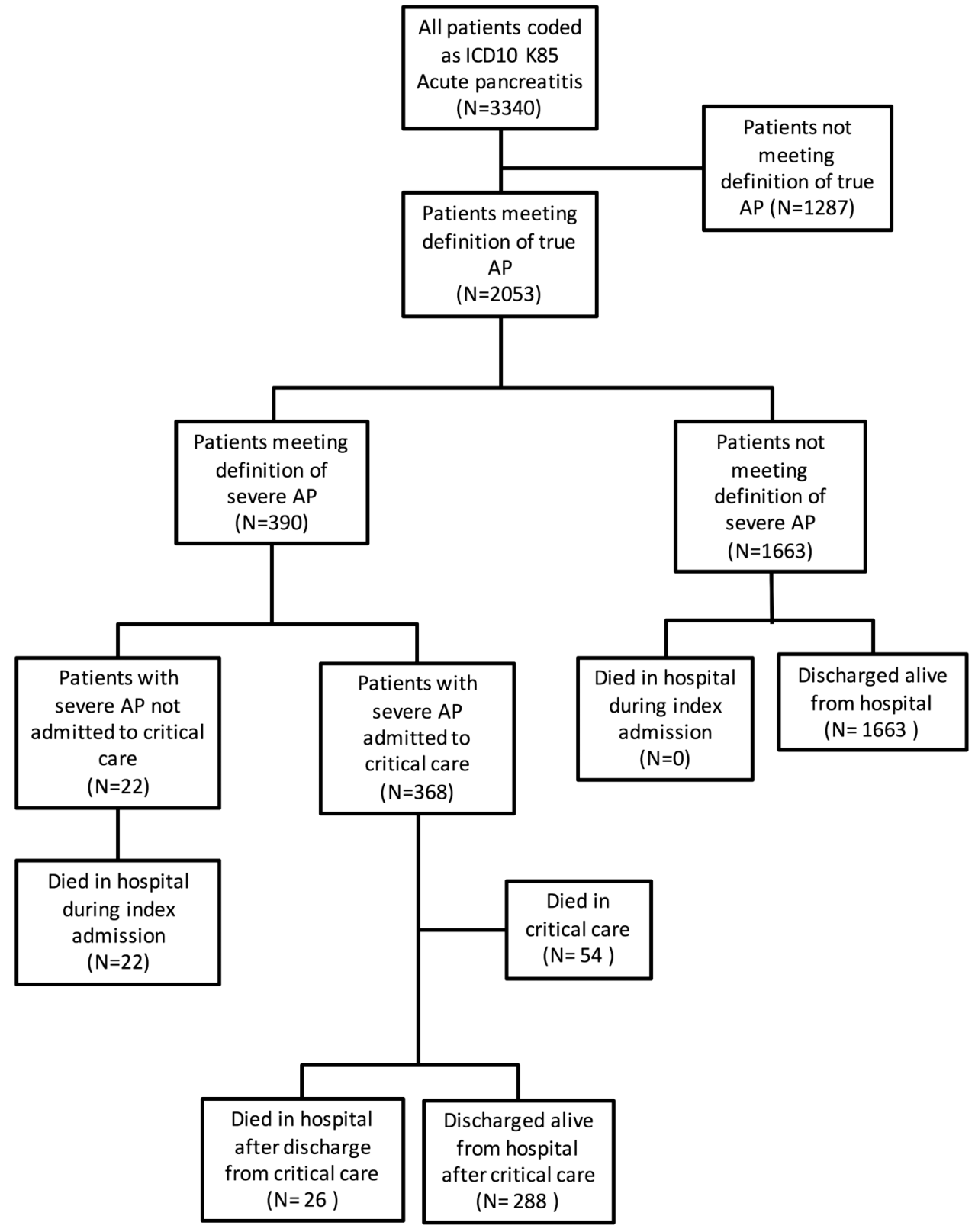

Figure 1 Flow diagram showing the fate of all potential participants in the study. AP, acute pancreatitis.

mechanical ventilation on the first admission, rising to $18.1 \%$ when all $\mathrm{CC}$ admissions for that $\mathrm{AP}$ episode were considered. $4.8 \%$ required invasive renal replacement therapy at any point during CC admission.

\section{Factors predicting SAP (admission to CC or death)}

The results of the logistic model predicting sAP in those with tAP are given in table 6. A lower calcium level $(-0.1 \mathrm{mmol} / \mathrm{L})$ at admission significantly increased the odds of developing sAP by $17 \%$ (OR 1.17 (95\% CI 1.08 to 1.26)). Patients previously admitted with AP were less likely to need CC admission during a subsequent admission (OR 0.77 (95\% CI 0.61 to 0.98 ) for one previous visit). A history of angina or IHD increased odds of sAP (OR 1.46 (95\% CI 1.07 to 2.00)), as did hypertension (OR 1.43 (95\% CI 1.05 to 1.96$)$ ), in addition to a primary care flag for alcohol dependency (OR 1.68 (95\% CI 0.83 to 3.41$)$ ). There was a significant interaction of age with type 2 diabetes. Generally, younger patients had a lower odds of sAP compared to those aged over 80 years, whereas for those aged 30-39 years with type 2 diabetes the odds were significantly increased (OR 18.85 (95\% CI 1.36 to 90.84)). The odds were still higher for those with type 2 diabetes as age increased but lower than for the 30-39 age group. The AUROC 0.68 and the Hosmer-Lemeshow test were non-significant.

\section{Mortality}

Overall in-hospital mortality was defined for patients with tAP only and was 102/2053 (5.0\%). Overall 90-day mortality for the tAP cohort was $127 / 2053(6.2 \%)$ and for the 
Table 1 Demographics and comorbidity of patients admitted with acute pancreatitis

\begin{tabular}{|c|c|c|c|c|c|c|c|}
\hline & $\begin{array}{l}\text { Patients coded } \\
\text { as ICD10 K85 } \\
\text { (AP) }\end{array}$ & $\begin{array}{l}\text { Patients meeting } \\
\text { definition of tAP }\end{array}$ & $\begin{array}{l}\text { Patients } \\
\text { meeting } \\
\text { definition } \\
\text { of SAP }\end{array}$ & $\begin{array}{l}\text { Patients with sAP } \\
\text { admitted to CC } \\
\text { (a subset of sAP) }\end{array}$ & $\begin{array}{l}\text { Patients with sAP not } \\
\text { admitted to critical } \\
\text { care (a subset of SAP } \\
\text { that died without CC } \\
\text { admission) }\end{array}$ & $\begin{array}{l}\text { Patients not } \\
\text { meeting definition } \\
\text { of sAP (nsAP) }\end{array}$ & $\begin{array}{l}\text { Comparison of nsAP } \\
\text { versus sAP (statistically } \\
\text { significant, } p \text { value; } \chi^{2} \\
\text { test } 1 \mathrm{df} \text {, unless } \\
\text { specified) }\end{array}$ \\
\hline \multicolumn{8}{|l|}{ Number of cases } \\
\hline ( $\mathrm{N}, \%$ total cases) & 3340 & $2053(61.5)$ & $390(11.7)$ & $368(11.0)$ & $22(0.7)$ & $1663(49.8)$ & \\
\hline$(\mathrm{N}, \% \mathrm{tAP})$ & & & $390(19.0)$ & 368 (17.9) & $22(1.1)$ & $1663(81.0)$ & \\
\hline$(\mathrm{N}, \% \mathrm{sAP})$ & & & & $368(94.4)$ & $22(5.6)$ & & \\
\hline \multicolumn{8}{|l|}{ Gender } \\
\hline $\begin{array}{l}\text { ( } \mathrm{N} \text { female, \% cases } \\
\text { within category) }\end{array}$ & $1578(47.2)$ & $1028(50.1)$ & $183(46.9)$ & $172(46.7)$ & $11(50)$ & $845(50.8)$ & 0.167 \\
\hline \multicolumn{8}{|l|}{ Age in years } \\
\hline Mean (SD) & $54.9(18.5)$ & $55.8(18.5)$ & $60.1(18.0)$ & $59.2(17.9)$ & $73.5(13.5)$ & $54.8(18.5)$ & $<0.001$ \\
\hline \multicolumn{8}{|l|}{ Body mass index $(\mathrm{N}=1466)$} \\
\hline Median (1st, 3rd quartile) & $26.7(22.7,31.6)$ & $27.5(23.0,32.3)$ & $28.4(23.3,33.1)$ & $28.4(24.1,33.1)$ & $22.1(18.9,30.7)$ & $27.3(22.9,32.0)$ & 0.227 \\
\hline \multicolumn{8}{|c|}{ Proxies for lifestyle (from GP records) } \\
\hline $\begin{array}{l}\text { ( } \mathrm{N} \text { for which this recorded, \% } \\
\text { total cases) }\end{array}$ & $1657(49.6)$ & $1039(50.6)$ & $195(50.0)$ & $185(50.3)$ & $10(45.6)$ & $844(50.8)$ & \\
\hline Overweight & $238(14.4)$ & $145(14.0)$ & $47(24.1)$ & $43(23.2)$ & $4(40.0)$ & $98(11.6)$ & $<0.001$ \\
\hline Prior/known alcohol overuse & $64(3.9)$ & $33(3.2)$ & $13(6.7)$ & $12(6.5)$ & $1(10.0)$ & $20(2.4)$ & 0.002 \\
\hline Smoking & $615(37.1)$ & $364(35.0)$ & $85(43.6)$ & $80(43.2)$ & $5(50.0)$ & $279(33.1)$ & 0.006 \\
\hline \multicolumn{8}{|l|}{ Aetiology of AP } \\
\hline ( $\mathrm{N}$ aetiology recorded, \%) & $3340(100)$ & $2053(100)$ & $390(100.0)$ & $368(100)$ & $22(100)$ & $1663(100)$ & \\
\hline $\begin{array}{l}\text { Gallstones (N, \% of } \\
\text { recorded) }\end{array}$ & $1482(44.4)$ & $919(44.8)$ & $171(43.9)$ & $165(44.8)$ & $6(27.3)$ & $748(45.0)$ & 0.686 \\
\hline $\begin{array}{l}\text { Alcohol+other non-gallstone } \\
\text { ( } \mathrm{N}, \% \text { of recorded) }\end{array}$ & $1877(55.6)$ & $1134(55.3)$ & $219(56.1)$ & $203(55.2)$ & $16(72.7)$ & $915(55.0)$ & \\
\hline \multicolumn{8}{|l|}{ Social deprivation quintile } \\
\hline Most deprived 1 & $1209(36.4 \%)$ & $720(35.3 \%)$ & $136(35 \%)$ & $128(34.9 \%)$ & & $584(35.4 \%)$ & Between groups \\
\hline \multicolumn{8}{|l|}{ (N, \% within category) } \\
\hline 2 & $704(21.2)$ & $412(20.2)$ & $79(20.3)$ & $72(19.6)$ & & $333(20.2)$ & $0.971\left(\chi^{2}\right.$ test $1 \mathrm{df}$ trend $)$ \\
\hline 3 & $543(16.3)$ & $327(16)$ & $66(17)$ & $65(17.7)$ & & $261(15.8)$ & \\
\hline 4 & $442(13.3)$ & $283(13.9)$ & $51(13.1)$ & $48(13.1)$ & & $232(14.0)$ & \\
\hline Most affluent 5 & $424(12.8)$ & $299(14.6)$ & $57(14.7)$ & $54(14.7)$ & & $242(14.6)$ & \\
\hline \multicolumn{8}{|c|}{ Duration of continuous inpatient stay, days } \\
\hline Median (1st, 3rd quartile) & $5(3,11)$ & $6(3,11)$ & $16(9,31)$ & $16(10,32)$ & $6(2,20)$ & $5(3,8)$ & <0.001 (Wilcoxon) \\
\hline
\end{tabular}

Bold typeface signifies $p<0.05$

*Exact proportion of alcohol diagnosis not possible for the entire cohort-see text for discussion.

AP, acute pancreatitis; CC, critical care; nsAP, not meeting definition of SAP; sAP, severe AP; tAP, true AP. 
Table 2 Healthcare prior to index AP admission

\begin{tabular}{|c|c|c|c|c|c|c|c|}
\hline & $\begin{array}{l}\text { Patients } \\
\text { coded as } \\
\text { ICD10 K85 } \\
\text { (AP) }\end{array}$ & $\begin{array}{l}\text { Patients } \\
\text { meeting the } \\
\text { definition of } \\
\text { tAP }\end{array}$ & $\begin{array}{l}\text { Patients } \\
\text { meeting the } \\
\text { definition of } \\
\text { SAP }\end{array}$ & $\begin{array}{l}\text { Patients with } \\
\text { sAP admitted } \\
\text { to CC } \\
\text { (sAP-CC) }\end{array}$ & $\begin{array}{l}\text { Patients with } \\
\text { sAP not } \\
\text { admitted to } \\
\text { CC }\end{array}$ & $\begin{array}{l}\text { Patients not } \\
\text { meeting the } \\
\text { definition of SAP } \\
\text { (nsAP) }\end{array}$ & $\begin{array}{l}\text { Comparison of nsAP } \\
\text { versus sAP (statistically } \\
\text { significant, } p \text { value; } \chi^{2} \text { test } 1 \mathrm{df} \text {, } \\
\text { unless specified) }\end{array}$ \\
\hline \multicolumn{8}{|l|}{ Number of cases } \\
\hline (N, \% total cases) & 3340 & $2053(61.5)$ & $390(11.7)$ & $368(11.0)$ & $22(0.7)$ & $1663(49.8)$ & \\
\hline \multicolumn{8}{|c|}{ Patients attending GP in year prior to admission } \\
\hline (N, \% total cases) & $618(18.5)$ & $317(15.4)$ & $48(12.3)$ & $42(11.4)$ & $6(27.3)$ & $269(16.2)$ & \\
\hline \multicolumn{8}{|c|}{ Number of GP visits in year prior to admission (GP attendees only) } \\
\hline $\begin{array}{l}\text { Median (1st, 3rd } \\
\text { quartile) }\end{array}$ & $5(2,11)$ & $5(2,10)$ & $7(3,13)$ & $5(2,12)$ & $12(7,18)$ & $5(2,9)$ & 0.075 (Mann-Whitney U-test) \\
\hline \multicolumn{8}{|c|}{ A\&E attendances in year prior to admission } \\
\hline $\begin{array}{l}\text { Median (1st, 3rd } \\
\text { quartile) }\end{array}$ & $1(1,3)$ & $1(1,2)$ & $1(1,2)$ & $1(1,2)$ & $2(1,4)$ & $1(1,2)$ & 0.745 (Mann-Whitney U-test) \\
\hline \multicolumn{8}{|c|}{ Hospital inpatient stays in year prior to admission } \\
\hline $\begin{array}{l}\text { Median (1st, 3rd } \\
\text { quartile) }\end{array}$ & $1(0,2)$ & $1(0,3)$ & $1(0,2)$ & $1(0,2)$ & $2(0,4)$ & $1(0,2)$ & 0.119 (Mann-Whitney U-test) \\
\hline \multicolumn{8}{|c|}{ Hospital outpatient visits in year prior to admission } \\
\hline $\begin{array}{l}\text { Median (1st, 3rd } \\
\text { quartile) }\end{array}$ & $1(0,3)$ & $1(0,3)$ & $1(0,3)$ & $1(0,3)$ & $1(0,2)$ & $1(0,3)$ & 0.202 (Mann-Whitney U-test) \\
\hline \multicolumn{8}{|c|}{ Number of previous admissions with AP } \\
\hline $\begin{array}{l}\text { Median (1st, 3rd } \\
\text { quartile) }\end{array}$ & $2(1,3)$ & $1(1,2)$ & $1(1,2)$ & $1(1,3)$ & $1(1,2)$ & $1(1,3)$ & 0.045 (Mann-Whitney U-test) \\
\hline \multicolumn{8}{|c|}{ Previous diagnoses or admissions } \\
\hline $\begin{array}{l}\text { Acute pancreatitis } \\
\text { (N, \% within category) }\end{array}$ & \multicolumn{6}{|c|}{ ( $\mathrm{N}, \%$ within category) } & $<0.001$ \\
\hline Gallstones & $628(18.8)$ & $364(17.7)$ & $61(15.6)$ & $58(15.8)$ & 2 (13.6) & $303(18.2)$ & 0.230 \\
\hline $\begin{array}{l}\text { Alcohol-related } \\
\text { psychiatric admission }\end{array}$ & $757(22.7)$ & $338(16.5)$ & $59(15.1)$ & $56(15.2)$ & $3(13.6)$ & $279(16.8)$ & 0.429 \\
\hline Hypertension & $942(28.2)$ & $595(29.0)$ & $152(39.0)$ & $143(38.9)$ & $9(40.9)$ & $443(26.6)$ & $<0.001$ \\
\hline $\begin{array}{l}\text { Ischaemic heart } \\
\text { disease }\end{array}$ & $539(16.1)$ & 334 (16.3) & $102(26.2)$ & $95(25.8)$ & $7(31.8)$ & $232(14.0)$ & $<0.001$ \\
\hline Myocardial infarction & $223(6.7)$ & $146(7.1)$ & $48(12.3)$ & $45(12.2)$ & $3(13.6)$ & $98(5.9)$ & $<0.001$ \\
\hline Stroke or TIA & $226(6.8)$ & $139(6.8)$ & $41(10.5)$ & 38 (10.3) & $3(13.6)$ & $98(5.9)$ & 0.001 \\
\hline Hypercholesteraemia & $197(5.9)$ & $125(6.1)$ & $21(5.4)$ & $21(5.7)$ & 0 & $104(6.3)$ & 0.518 \\
\hline $\begin{array}{l}\text { DM type } 2 \text { not on } \\
\text { insulin }\end{array}$ & $471(14.1)$ & 267 (13.0) & 79 (20.3) & $79(21.5)$ & 0 & $188(11.3)$ & $<0.001$ \\
\hline $\begin{array}{l}\text { DM type } 2 \text { treated } \\
\text { with insulin }\end{array}$ & $57(1.7)$ & $20(1.0)$ & $6(1.5)$ & $5(1.4)$ & $1(4.5)$ & $14(0.8)$ & 0.208 \\
\hline DM type 1 & $113(3.4)$ & $45(2.2)$ & $9(2.3)$ & $9(2.4)$ & 0 & $36(2.2)$ & 0.862 \\
\hline DM (unspecified) & $124(3.7)$ & $66(3.2)$ & $19(4.9)$ & $18(4.9$ & $1(4.5)$ & $47(2.8)$ & 0.039 \\
\hline Asthma & $345(10.3)$ & $198(9.6)$ & $45(11.5)$ & $43(11.7)$ & $2(9.1)$ & $153(9.2)$ & 0.159 \\
\hline \multirow[t]{2}{*}{ COPD } & $313(9.4)$ & $180(8.8)$ & 47 (12.1) & 40 (10.9) & $7(31.8)$ & $133(8.0)$ & 0.011 \\
\hline & & & & & & & Cor \\
\hline
\end{tabular}




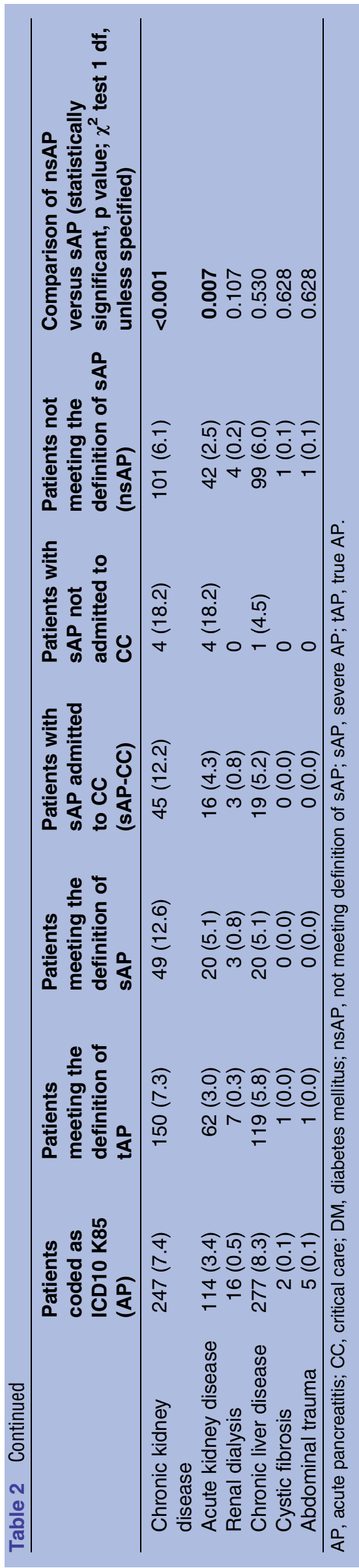

sAP cohort was 100/390 (25.6\%). In-hospital mortality among sAP admitted to CC was 80/368 (21.7\%), of whom 54/368 (14.7\%) died in CC and 26/368 (7.1\%) died in hospital after discharge from CC (table 5).

Patients who died in CC died after a median (1st, 3rd quartile) of 70 (25-371) hours after CC admission (table 5). The median time from hospital admission to death for those dying in CC was 82 (26 371) hours, from which it can be inferred that the median time between admission to hospital and admission to CC was 12 hours (table 5). Patients who died in hospital after discharge from CC died after a median (1st, 3rd quartile) of 9.9 (5.1-21.9) days and mean 23.0 days after CC admission.

Twenty-two patients with sAP died in hospital without being admitted to CC after a median (1st, 3rd quartile) of 4.5 (2.0-18.0) days and mean of 12.0 days. The specific reasons why patients with sAP in this subgroup were not admitted to CC are not known. None of these patients had advanced malignancy recorded as a cause or contributor on their death certificate. As a surrogate to identify nursing home residents who may not have been suitable for CC, we examined place of residence. All 22 were admitted from private residence rather than a nursing home and 2 of 22 lived alone. Since prehospital and early mortality in sAP is greater in rural areas, ${ }^{21}$ we examined postcodes for this patient subgroup. Of 22 patients, 20 were from urban postcodes. The mean \pm SD age of these 22 patients was $74 \pm 14$ years and median (range) was 72 (46-92) years. The median BMI (1st, 3rd quartile) for these 22 patients was significantly lower (22.1 (18.9-30.7)) than the respective measures for the remainder of the tAP cohort.

\section{Risk of death after CC admission}

The results of fitting the parametric Gamma model (lowest AIC) are provided in table 7 and figure 2. Generally, negative regression coefficients indicate factors that shorten the time to death. The only factor that was positive was T2 DM, which gave a time ratio of 2.99 (95\% CI 0.42 to 21.28$)$. This indicates that the time to death was threefold longer in those with T2 DM. There were very significant associations of shortening of time to death with thyroid/parathyroid history ( $\mathrm{TR}=0.003,95 \%$ CI 0.001 to 0.012 ) and history of coronary artery stent in previous year $(\mathrm{TR}=0.049,95 \%$ CI 0.014 to 0.168). For each unit increase in APACHE II score, there was a $29 \%$ reduction in time to death ( $\mathrm{TR}=0.71,95 \%$ CI 0.48 to 1.05 ), whereas for each additional prior $\mathrm{CC}$ admission, the time to death reduced by $34 \%$ (TR=0.66 95\% CI 0.37 to 1.19 ). Finally, for each increase in age by 1 year, the time to death reduced by $4 \%$ (TR $=0.9695 \%$ CI 0.92 to 1.01 ). Probabilities estimated from the Gamma model were very close to the Kaplan-Meier estimates of survival.

\section{DISCUSSION}

AP is associated with a high mortality, shortens overall life expectancy in survivors ${ }^{22}$ and constitutes a 


\begin{tabular}{|c|c|c|c|c|c|c|c|}
\hline & $\begin{array}{l}\text { Patients coded } \\
\text { as ICD10 K85 } \\
\text { (AP) }\end{array}$ & $\begin{array}{l}\text { Patients meeting } \\
\text { definition of } t A P\end{array}$ & $\begin{array}{l}\text { Patients } \\
\text { meeting } \\
\text { definition of } \\
\text { SAP }\end{array}$ & $\begin{array}{l}\text { Patients admitted } \\
\text { to CC (a subset of } \\
\text { SAP) }\end{array}$ & $\begin{array}{l}\text { Patients with sAP } \\
\text { not admitted to CC } \\
\text { (a subset of sAP) }\end{array}$ & $\begin{array}{l}\text { Patients not } \\
\text { meeting definition } \\
\text { of sAP (nsAP) }\end{array}$ & $\begin{array}{l}\chi^{2} \text { test of } \\
\text { sAP versus } \\
\text { nsAP } \\
\text { p Value }\end{array}$ \\
\hline \multicolumn{8}{|l|}{ Number of cases } \\
\hline (N, \% total cases) & 3340 & $2053(61.5)$ & $390(11.7)$ & $368(11.0)$ & $22(0.7)$ & $1663(49.8)$ & \\
\hline \multicolumn{8}{|c|}{ Prescribed drugs in year up to and including admission } \\
\hline Paracetamol & $1557(46.6)$ & $917(44.7)$ & $202(51.8)$ & $188(51.1)$ & 14 (63.6) & $715(43.0)$ & 0.001 \\
\hline \multicolumn{8}{|l|}{ ( $\mathrm{N}, \%$ within category) } \\
\hline Antidepressants & $544(16.3)$ & $304(14.8)$ & $67(17.2)$ & $64(17.4)$ & $3(13.6)$ & $237(14.3)$ & 0.137 \\
\hline Calcium-altering drugs & 466 (14) & $283(13.8)$ & $78(20)$ & $74(20.1)$ & $4(18.2)$ & 205 (12.3) & $<0.001$ \\
\hline Azathioprine & $26(0.8)$ & $17(0.8)$ & $4(1)$ & $4(1.1)$ & 0 & $13(0.8)$ & 0.629 \\
\hline Carbemazepine & $51(1.5)$ & $26(1.3)$ & $3(0.8)$ & $3(0.8)$ & 0 & $23(1.4)$ & 0.331 \\
\hline Oestrogens & $158(4.7)$ & $107(5.2)$ & $21(5.4)$ & $21(5.7)$ & 0 & $86(5.2)$ & 0.818 \\
\hline Neuroleptic agents & 876 (26.2) & $512(24.9)$ & $116(29.7)$ & $108(29.3)$ & $8(36.4)$ & $396(23.8)$ & 0.017 \\
\hline Oral antidiabetic agents & $247(7.4)$ & $144(7)$ & $33(8.5)$ & $32(8.7)$ & $1(4.5)$ & $111(6.7)$ & 0.209 \\
\hline Oral corticosteroids & $206(6.2)$ & $133(6.5)$ & $35(9)$ & $28(7.6)$ & $7(31.8)$ & 98 (5.9) & 0.025 \\
\hline Simvastatin & $625(18.7)$ & 402 (19.6) & $110(28.2)$ & $107(29.1)$ & $3(13.6)$ & $292(17.6)$ & $<0.001$ \\
\hline $\begin{array}{l}\text { Statins (excluding } \\
\text { simvastatin) }\end{array}$ & $56(1.7)$ & $35(1.7)$ & $11(2.8)$ & $10(2.7)$ & $1(4.5)$ & $24(1.4)$ & 0.058 \\
\hline \multicolumn{8}{|c|}{ Procedures in year up to and including admission } \\
\hline ERCP & $267(8.0)$ & $115(5.6)$ & $18(4.6)$ & $18(4.9)$ & 0 & $97(5.8)$ & 0.352 \\
\hline \multicolumn{8}{|l|}{ ( $\mathrm{N}, \%$ within category) } \\
\hline Cholecystectomy & $254(7.6)$ & $142(6.9)$ & $19(4.9)$ & $18(4.9)$ & $1(4.5)$ & $123(7.4)$ & 0.079 \\
\hline $\begin{array}{l}\text { Thyroid/parathyroid } \\
\text { surgery }\end{array}$ & $13(0.4)$ & $8(0.4)$ & $1(0.3)$ & $1(0.3)$ & 0 & $7(0.4)$ & 0.640 \\
\hline Coronary artery stent & $40(1.2)$ & $27(1.3)$ & $11(2.8)$ & $11(3.0)$ & 0 & $16(1.0)$ & 0.004 \\
\hline Heart valve and/or & $15(0.4)$ & $9(0.4)$ & $1(0.3)$ & $1(0.3)$ & 0 & $8(0.5)$ & 0.547 \\
\hline CABG & & & & & & & \\
\hline \multicolumn{8}{|c|}{$\begin{array}{l}\text { AP, acute pancreatitis; CABG, coronary artery bypass grafting; CC, critical care; ERCP: endoscopic retrograde cholangiopancreatography; nsAP, not meeting definition of sAP; sAP, severe AP; } \\
\text { tAP, true AP. }\end{array}$} \\
\hline
\end{tabular}

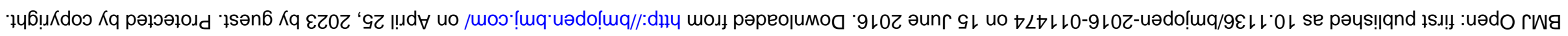


Table 4 Admission to critical care

\begin{tabular}{|c|c|c|}
\hline & First CC admission & All CC admissions \\
\hline \multicolumn{3}{|c|}{ CC admissions during one continuous inpatient stay } \\
\hline 1 or more & & $368(100 \%)$ \\
\hline 2 or more & & $101(27.4 \%)$ \\
\hline 3 or more & & $23(6.2 \%)$ \\
\hline 4 or more & & $5(1.4 \%)$ \\
\hline 5 & & $1(0.0 \%)$ \\
\hline \multicolumn{3}{|c|}{ Days from hospital admission to first CC admission } \\
\hline Mean $(95 \% \mathrm{Cl})$ & $3.03(2.32$ to 3.74$)$ & \\
\hline $5 \%$ trimmed mean & 1.83 & \\
\hline Median (1st, 3rd quartile) & $1(0,2)$ & \\
\hline \multicolumn{3}{|l|}{ APACHE II score at CC admission } \\
\hline Median (1st, 3rd quartile) & $19(14,24)$ & $19(14,24)$ \\
\hline \multicolumn{3}{|c|}{ Organs requiring invasive support at CC admission } \\
\hline 0 & $123(33.4 \%)$ & $139(27.9 \%)$ \\
\hline 1 & $124(33.7 \%)$ & $162(32.5 \%)$ \\
\hline 2 & $96(26.1 \%)$ & $157(31.5 \%)$ \\
\hline 3 & $22(6 \%)$ & $36(7.2 \%)$ \\
\hline 4 & $3(0.8 \%)$ & $4(0.8 \%)$ \\
\hline \multicolumn{3}{|l|}{ Organ support required at CC admission } \\
\hline No immediate organ support given & $24(6.5 \%)$ & $26(5.2 \%)$ \\
\hline \multicolumn{3}{|l|}{ Respiratory } \\
\hline Facemask/nasal cannulae & $296(80.4 \%)$ & $382(76.7 \%)$ \\
\hline $\mathrm{O}_{2}$ less than $50 \%$ & $206(56.0 \%)$ & $254(51)$ \\
\hline $\mathrm{O}_{2} 50 \%$ or more & 77 (20.9\%) & $111(22.3 \%)$ \\
\hline Continuous positive airway pressure & $10(2.7 \%)$ & $17(3.4 \%)$ \\
\hline Endotracheal tube & $46(12.5 \%)$ & $85(17.1 \%)$ \\
\hline Mechanical ventilator & $51(13.9 \%)$ & $90(18.1 \%)$ \\
\hline Tracheostomy & $2(0.5 \%)$ & $5(1 \%)$ \\
\hline \multicolumn{3}{|l|}{ Cardiovascular } \\
\hline Central venous catheter & $136(37.0 \%)$ & $217(43.6 \%)$ \\
\hline Arterial line & $186(50.5 \%)$ & $276(55.4 \%)$ \\
\hline Inotropes/vasopressors & $222(60.3 \%)$ & $326(65.5 \%)$ \\
\hline Single vasoactive/antiarrhythmic drugs & $37(10.5 \%)$ & $66(13.3 \%)$ \\
\hline Multiple vasoactive/antiarrhythmic drugs & $15(4.2 \%)$ & $21(4.2 \%)$ \\
\hline Invasive cardiac output monitoring & $10(2.8 \%)$ & $16(3.2 \%)$ \\
\hline Pulmonary artery catheter & $0(0 \%)$ & $0(0 \%)$ \\
\hline Oesophageal Doppler waveform analysis & $1(0.3 \%)$ & $3(0.6 \%)$ \\
\hline Peripheral pulse contour analysis & $8(2.2 \%)$ & $10(2)$ \\
\hline Other method & $1(0.3 \%)$ & $3(0.6 \%)$ \\
\hline \multicolumn{3}{|l|}{ Renal } \\
\hline Haemofiltration/dialysis & $17(4.6 \%)$ & $24(4.8 \%)$ \\
\hline \multicolumn{3}{|l|}{ Neurological } \\
\hline Invasive neurological monitoring & $0(0 \%)$ & $0(0 \%)$ \\
\hline
\end{tabular}

considerable healthcare resource burden. $^{7-9} 13$ Specifically, the evolution of persistent organ dysfunction requiring $\mathrm{CG}$ support has the most deleterious short-term and long-term consequences in AP. Building on previous work by others, ${ }^{23} 24$ in this cross-sectional observational study, we have brought together 16 electronic healthcare records into a single national Safe Haven overseen by the Farr Institute governance structure to conduct a detailed study of the development of organ dysfunction and CC admission in AP.

These data confirm a disproportionately high incidence of AP in the most multiply deprived quintiles of areas, in keeping with previous observations by us and others, ${ }^{25}$ and that CG admission during an episode of AP has a high $(21.7 \%)$ mortality compared to that of the overall cohort $(5.0 \%)$. Patients who require CC admission do so early in the disease course (at a median of 1 day after admission) and experience a median total of 6 days CC support per episode of AP. Factors independently predictive of $\mathrm{CC}$ admission or death include a history of angina, pre-existing hypertension, hypocalcaemia, fewer prior emergency admissions with AP and type 2 diabetes in young patients. The reasons for the association with young patients with type 2 diabetes has not 
been ascertained from our data, despite our efforts to do so; it is possible that this observation may reflect differences in older persons, or reflect a confounder in our data set that we have been unable to uncover. Once admitted to CC, factors predictive of death included cardiovascular stenting and thyroid or parathyroid surgery within the previous year.

The overall mortality seen in our study is comparable with that seen in other large epidemiological studies in the UK, ${ }^{26}$ the USA, ${ }^{7}{ }^{8}$ Taiwan, ${ }^{2}$ Japan $^{4}$ and South Africa. ${ }^{27}$ The mortality in the population of patients with tAP requiring $\mathrm{CC}$ in the present cohort is somewhat
All CC periods

Sum of CC periods in one continuous inpatient stay

368
$54(14.7 \%)$
$70(25,371)$
260
$82(26371)$
264
$107(45,221)$
179
$6(3,11)$
8.76
$4(2,7)$
5.43
$0(0,0)$
$6(3,8)$ on dialysis
1.30
8.1 on dialysis

$9.8(8.2,11.2)$

$15.4(11.4,23.6)$

$5.3(4.5,6.1)$

$5.7(5.0,6.7)$

$7.28(7.20,7.37)$

$7.36(7.27,7.42)$

$90(60,100)$

$144(80,272)$

$25(14,58)$ 
Table 6 Logistic regression of factors predicting the development of tAP to SAP (CC admission or death)

\begin{tabular}{|c|c|c|}
\hline Parameter & $\begin{array}{l}\text { Regression } \\
\text { estimate* }^{*}\end{array}$ & SE \\
\hline Intercept & 2.499 & 1.069 \\
\hline T2 DM no insulin (yes/no) & -0.298 & 0.442 \\
\hline $\begin{array}{l}\text { Simvastatin } 12 \text { months prior } \\
\text { (yes/no) }\end{array}$ & 0.221 & 0.151 \\
\hline $\begin{array}{l}\text { Number of prior AP emergency } \\
\text { admission ( }+1 \text { admission) }\end{array}$ & -0.259 & 0.123 \\
\hline Angina or IHD (yes/no) & 0.379 & 0.161 \\
\hline Hypertension (yes/no) & 0.360 & 0.161 \\
\hline $\begin{array}{l}\text { Test result calcium } \dagger \text { at index } \\
\text { admission }(-0.1 \mathrm{mmol} / \mathrm{L})\end{array}$ & 0.156 & 0.038 \\
\hline \multicolumn{3}{|l|}{ Age group (vs $80+$ ) } \\
\hline $18-19$ & -0.386 & 0.780 \\
\hline 20-29 & -0.867 & 0.630 \\
\hline 20-29† T2 DM (yes/no) & 1.235 & 1.279 \\
\hline 30-39 & -0.879 & 0.614 \\
\hline 30-39† T2 DM (yes/no) & 2.936 & 0.802 \\
\hline $40-49$ & 1.053 & 0.608 \\
\hline 40-49† T2 (yes/no) & 0.643 & 0.686 \\
\hline $50-59$ & -0.342 & 0.596 \\
\hline 50-59† T2 (yes/no) & 0.292 & 0.576 \\
\hline $60-79$ & -0.466 & 0.591 \\
\hline 60-79† T2 (yes/no) & 0.890 & 0.487 \\
\hline Alcohol flag† (yes/no) & 0.521 & 0.360 \\
\hline \multicolumn{3}{|c|}{$\begin{array}{l}\text { *Increase in logodds. } \\
+19 \% \text { missing calcium, } 35 \% \text { missing alcohol; } 98 \% \text { relative } \\
\text { efficiency of multiple imputations with } 10 \text { imputations. } \\
\text { AP, acute pancreatitis; CC, critical care; DM, diabetes mellitus; } \\
\text { nsAP, not meeting definition of SAP; SAP, severe AP; tAP, true } \\
\text { AP. }\end{array}$} \\
\hline
\end{tabular}

that it is possible that a faster evolution variant of $\mathrm{AP}$ is more lethal, or that this subgroup of patients is more susceptible to rapid evolution of disease. Finally, this observation may reflect a delay in presentation to hospital leading to increased mortality in those not presenting promptly for treatment.

Independent predictors of severe disease in tAP included a history of angina and hypertension. It is logical that patients with pre-existing chronic heart

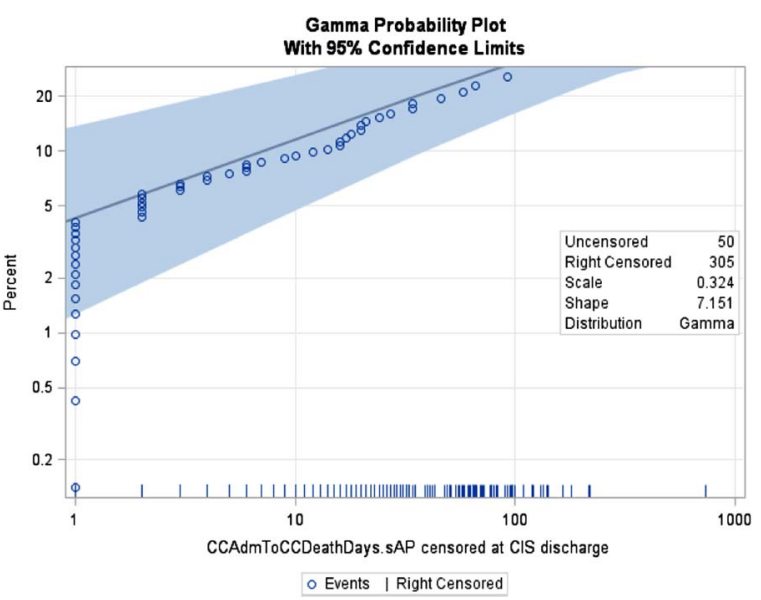

Figure 2 Probability plot (gamma model) of risk factors for death after admission to critical care. CC, critical care; SAP, severe acute pancreatitis.

disease would be at increased risk of death once they develop organ dysfunction, and this is borne out in our study by the association between coronary stenting in the preceding year and risk of death after CC admission. However, it is not as straightforward to link angina and hypertension with an increased likelihood of needing CC admission in the context of an AP episode. Angina and hypertension in this scenario may be confounders, acting as surrogate markers of other chronic health conditions that directly reduce the threshold for developing organ dysfunction requiring CC, or they may indicate a lack of physiological compensatory reserve in critical illness, such that for a given inflammatory insult there is greater physiological derangement.

Our observation that a higher number of previous admissions with AP reduces the risk for $\mathrm{CC}$ admission or death during a subsequent AP episode is novel. It is uncertain whether this is a result of increased individual likelihood of developing systemic organ dysfunction with particular severity of inflammatory insult, or whether frequent A\&E attenders, particularly with regard to alcohol overuse, represent a different subset of patients.

Table 7 Factors that impact on time to death following CC from a Gamma prediction model

\begin{tabular}{|c|c|c|c|c|c|c|}
\hline \multirow{2}{*}{$\frac{\text { Parameter }}{\text { Intercept }}$} & \multirow{2}{*}{$\begin{array}{l}\begin{array}{l}\text { Regression } \\
\text { estimate* }\end{array} \\
10.006\end{array}$} & \multirow{2}{*}{$\begin{array}{l}\text { SE } \\
1.752\end{array}$} & \multicolumn{2}{|c|}{$\begin{array}{l}95 \% \text { Confidence } \\
\text { limits }\end{array}$} & \multirow{2}{*}{$\begin{array}{l}\chi^{2} \\
32.61\end{array}$} & \multirow{2}{*}{$\begin{array}{l}\mathrm{p} \text { Value } \\
<0.0001\end{array}$} \\
\hline & & & 6.572 & 13.440 & & \\
\hline Age at index admission ( +1 year) & -0.037 & 0.022 & -0.080 & 0.006 & 2.83 & 0.093 \\
\hline T2 DM (yes/no) & 1.095 & 1.001 & -0.867 & 3.058 & 1.20 & 0.274 \\
\hline Log (APACHE II) (+1 unit) & -0.345 & 0.200 & -0.736 & 0.046 & 2.99 & 0.084 \\
\hline $\begin{array}{l}\text { Coronary artery stent within } \\
1 \text { year (yes/no) }\end{array}$ & -3.015 & 0.628 & -4.245 & -1.785 & 23.08 & $<0.0001$ \\
\hline Thyroid/parathyroid (yes/no) & -5.875 & 0.746 & -7.336 & -4.414 & 62.10 & $<0.0001$ \\
\hline $\begin{array}{l}\text { Number of CC stays in previous year } \\
\text { ( }+1 \text { CC stay) }\end{array}$ & -0.414 & 0.299 & -1.000 & 0.172 & 1.92 & 0.166 \\
\hline Gamma scale parameter & 0.326 & 0.053 & 0.238 & 0.448 & & \\
\hline Gamma shape parameter & 6.816 & 1.093 & 4.673 & 8.958 & & \\
\hline
\end{tabular}

${ }^{*}$ Log of time to death ratio (negative represents a shorter time to death).

$\mathrm{CC}$, critical care; DM, diabetes mellitus. 
The relationship between fluctuations in serum calcium and pancreatitis is $\mathrm{known}^{29}$ and the association between thyroid and parathyroid surgery and death after CC admission and between hypocalcaemia and calcium-altering drugs is not surprising. Hypocalcaemia or hypercalcaemia is recognised in the Imrie $^{30}$ and Ranson $^{31}$ scoring systems, and there is a wealth of preclinical experimental evidence of deranged calcium signalling as a mechanism of pancreatic acinar cell injury. ${ }^{32}$

\section{Strengths of study}

The main strength of this study is the coalition of multiple electronic data sources linked though a unique identifier on a national basis. Critical to this process was strict data protection. This was achieved through the use of a centralised protected safe haven and minimising the number of individuals with access to the non-anonymised primary data. Furthermore, industry partners could be fully involved in the protocol design and processes surrounding the data collection and depository without needing sight of any primary data. Combining detailed inpatient CC information collected at high temporal resolution and integrating this with broad background data derived from community data sources were achieved.

\section{Limitations and bias}

Limitations of this study include an inability to gain information in electronic form on routinely recorded clinical data from those inpatients who did not require CC. This may have led to a negative reporting bias for events that might be relevant to this study but not sufficiently severe to necessitate CC admission. In addition, we are unable to ascertain exactly how many individuals might have met criteria for CC admission but not admitted due to advance directives or limited capacity. A further limitation inherent to the study design based on routinely collected data is the relative lack of confirmation of the accuracy of source data entry. Although we specifically conducted a coding accuracy pilot for the diagnosis of AP, comparing electronic records with hand-written notes in four separate study sites, and while certain variables are likely to be highly accurate, for example automatically retrieved Wardwatcher critical care data, laboratory values and date of death on death certificates, certain other fields are impossible to ratify accurately. We include in the latter category comorbidity and clinical information recorded in hospital discharge summaries.

\section{CONCLUSIONS}

National record-linkage analyses from a wide variety of routinely collected primary and secondary care data sources are feasible and constitute a powerful resource. Factors that predict sAP are distinct from those that prognosticate mortality. Mortality in patients with AP who require $\mathrm{CC}$ admission remains high.
Author affiliations

${ }^{1}$ MRC Centre for Inflammation Research, Queen's Medical Research Institute, University of Edinburgh, Edinburgh, UK

${ }^{2}$ Clinical Surgery, University of Edinburgh, Edinburgh, UK

${ }^{3}$ Healthcare Information Factory, Observational Data Analytics Group, Worldwide Epidemiology, GSK, Uxbridge, UK

${ }^{4}$ eDRIS, NHS National Services Scotland, Edinburgh, UK

${ }^{5}$ Clinical Statistics Consultants, Dundee, UK

${ }^{6}$ Farr Institute, Edinburgh, UK

${ }^{7}$ Department of Family and Community Medicine, North York General Hospital, University of Toronto, Toronto, Ontario, Canada

${ }^{8}$ Dundee Epidemiology and Biostatistics Unit (DEBU), Division of Population Health Sciences, Medical Research Institute, University of Dundee, Dundee, UK

Acknowledgements The authors acknowledge the valuable contributions of the following: Jacqueline Caldwell, Farr at Scotland; Duncan Heather, Health Informatics Centre Farr Dundee; Roma Armstrong, Research and Development Management Office, NHS Greater Glasgow and Clyde, UK; Ron Jamieson, lay member of the Project Board; colleagues in NHS Information Services, Division and the Department of Surgery, University of Edinburgh; lain Uings, Nicholas Galwey, George Dukes and John Liddle, GSK; DJM gratefully acknowledges the support of the Health Foundation/Academy of Medical Sciences through the Clinician Scientist Fellowship scheme.

Contributors DJM contributed to the study idea, protocol design, literature search, limited data analysis, drafted manuscript, figures and tables, research in context and cover letter. UG contributed to the study idea, protocol design, literature search, limited data analysis, commented on and revised drafts of the manuscript, figures and tables, research in context and cover letter. PJ contributed to the study idea, protocol design, pilot data, safe haven, coordinated the study data linkage and conduct and commented on and revised drafts of the manuscript, figures and tables. LC performed the statistical analysis and contributed to, commented on and revised drafts of the manuscript, figures and tables. LH contributed to the study idea, protocol design, pilot data, contributed to the study data linkage and conduct and commented on and revised drafts of the manuscript, figures and tables. GMAW contributed to the protocol design, study data linkage, safe haven design, and conduct and commented on and revised drafts of the manuscript, figures and tables. CS contributed to the literature search and revised and commented on the manuscript, figures and tables, research in context and cover letter. CD contributed to the study data linkage, safe haven design, and conduct and commented on and revised drafts of the manuscript, figures and tables. FS contributed to the study idea, protocol design, pilot data, safe haven, contributed to the design of the study data linkage, and conduct and commented on and revised drafts of the manuscript, figures and tables. $\mathrm{AM}$ contributed to the study idea and commented on and revised drafts of the manuscript, figures and tables. HJTW contributed to the study idea, protocol design, pilot data, safe haven, coordinated the study data linkage, and conduct and commented on and revised drafts of the manuscript, figures and tables. AML contributed to the study idea, protocol design, literature search, commented on data analysis and contributed to revising the manuscript, figures and tables, research in context and cover letter. PTD contributed to the study idea, protocol design, performed data analysis and statistical modelling, drafted sections of the manuscript, figures and tables, and commented on and revised on all sections and the research in context and cover letter.

Funding This study was commissioned by GSK through the Farr Institute/ SHIP/eDRIS single portal. DJM is a Clinician Scientist Fellow funded by the Health Foundation/Academy of Medical Sciences.

Competing interests The University of Edinburgh collaborates with GSK through a Discovery Partnership with Academia agreement. PTD is a member of the New Drugs Committee of the Scottish Medicines Consortium. There are no other conflicts of interest to declare.

Provenance and peer review Not commissioned; externally peer reviewed.

Data sharing statement Applications for data for this study should be made to the Farr Institute/NHS ISD/eDRIS single portal, via an initial approach to the corresponding author. No additional data are available outwith that process. 
Open Access This is an Open Access article distributed in accordance with the Creative Commons Attribution Non Commercial (CC BY-NC 4.0) license, which permits others to distribute, remix, adapt, build upon this work noncommercially, and license their derivative works on different terms, provided the original work is properly cited and the use is non-commercial. See: http:// creativecommons.org/licenses/by-nc/4.0/

\section{REFERENCES}

1. Goldacre MJ, Roberts SE. Hospital admission for acute pancreatitis in an English population, 1963-98: database study of incidence and mortality. BMJ 2004;328:1466-9.

2. Shen HN, Lu CL, Li CY. Epidemiology of first-attack acute pancreatitis in Taiwan from 2000 through 2009: a nationwide population-based study. Pancreas 2012;41:696-702.

3. Omdal T, Dale J, Lie SA, et al. Time trends in incidence, etiology, and case fatality rate of the first attack of acute pancreatitis. Scand $J$ Gastroenterol 2011;46:1389-98.

4. Satoh K, Shimosegawa T, Masamune A, et al. Nationwide epidemiological survey of acute pancreatitis in Japan. Pancreas 2011;40:503-7.

5. Fagenholz PJ, Castillo CF, Harris NS, et al. Increasing United States hospital admissions for acute pancreatitis, 1988-2003. Ann Epidemiol 2007;17:491-7.

6. Roberts SE, Thorne K, Evans PA, et al. Mortality following acute pancreatitis: social deprivation, hospital size and time of admission: record linkage study. BMC Gastroenterol 2014;14:153.

7. Peery AF, Dellon ES, Lund J, et al. Burden of gastrointestinal disease in the United States: 2012 update. Gastroenterology 2012;143:1179-87.e1-3.

8. Everhart JE, Ruhl CE. Burden of digestive diseases in the United States Part III: liver, biliary tract, and pancreas. Gastroenterology 2009;136:1134-44.

9. Mole DJ, Haddow C, Cook B, et al. Critical care resource use and mortality from severe acute pancreatitis in Scotland, 2008 to 2012. Oxford: HPB, 2012: 633

10. Forsmark CE, Baillie J. AGA Institute technical review on acute pancreatitis. Gastroenterology 2007;132:2022-44.

11. Banks PA, Bollen TL, Dervenis C, et al. Classification of acute pancreatitis-2012: revision of the Atlanta classification and definitions by international consensus. Gut 2013;62:102-11.

12. McKay CJ, Imrie CW. The continuing challenge of early mortality in acute pancreatitis. Br J Surg 2004;91:1243-4

13. Mole D, Garden OJ, Iredale J. Alcohol and global health: focus on acute pancreatitis needed. Lancet 2009;374:976-7; author reply 77 http://dx.doi.org/10.1016/S0140-6736(09)61659-7

14. Farr Institute. http://www.farrinstitute.org

15. Mole D, Webster S, Uings I, et al. Kynurenine-3-monooxygenase inhibition prevents multiple organ failure in rodent models of acute pancreatitis. Nat Med 2016;22:202-9.
16. Scottish Government. Scottish index of multiple deprivation 2012: a National Statistics Publication for Scotland. Edinburgh, 2012.

17. Kendrick S, Clarke J. The Scottish record linkage system. Health Bull (Edinb) 1993;51:72-9.

18. Howe GR, Spasoff RA, eds. Computerised record linkage in health research: an overview. Proceedings of the Workshop on Computerised Linkage in Health Research; Ottowa, Ontario, Canada. Toronto: University of Toronto Express, 1986.

19. SHIP. A blueprint for health records research in Scotland. Edinburgh, 2012. http://www.scot-ship.ac.uk/sites/default/files/ Reports/SHIP_BLUEPRINT_DOCUMENT_final_100712.pdf

20. von Elm E, Altman DG, Egger M, et al. The Strengthening the Reporting of Observational Studies in Epidemiology (STROBE) statement: guidelines for reporting observational studies. Lancet 2007;370:1453-7.

21. Andersson R, Andren-Sandberg A. Fatal acute pancreatitis Characteristics of patients never reaching hospital. Pancreatology 2003;3:64-6.

22. Skouras C, Hayes AJ, Williams L, et al. Early organ dysfunction affects long-term survival in acute pancreatitis patients. Oxford: HPB, 2014.

23. McKay CJ, Evans S, Sinclair M, et al. High early mortality rate from acute pancreatitis in Scotland, 1984-1995. Br J Surg 1999;86:1302-5.

24. Wilson $\mathrm{C}$, Imrie CW. Changing patterns of incidence and mortality from acute pancreatitis in Scotland, 1961-1985. Br J Surg 1990;77:731-4.

25. Ellis MP, French JJ, Charnley RM. Acute pancreatitis and the influence of socioeconomic deprivation. Br J Surg 2009;96:74-80.

26. Roberts SE, Williams JG, Meddings D, et al. Incidence and case fatality for acute pancreatitis in England: geographical variation, social deprivation, alcohol consumption and aetiology -a record linkage study. Aliment Pharmacol Ther 2008;28:931-41.

27. John KD, Segal I, Hassan $\mathrm{H}$, et al. Acute pancreatitis in Sowetan Africans. A disease with high mortality and morbidity. Int $J$ Pancreatol 1997;21:149-55.

28. SICSAG. Scottish Intensive Care Society Audit Group. 2012. http:// www.sicsag.scot.nhs.uk/publications/main.htm (accessed 9 Jun 2016).

29. Gerasimenko JV, Gerasimenko OV, Petersen $\mathrm{OH}$. The role of $\mathrm{Ca}^{2+}$ in the pathophysiology of pancreatitis. J Physiol (Lond) 2014;592 (Pt 2):269-80.

30. Imrie CW, Benjamin IS, Ferguson JC, et al. A single-centre double-blind trial of Trasylol therapy in primary acute pancreatitis. Br J Surg 1978;65:337-41.

31. Ranson JH, Rifkind KM, Roses DF, et al. Prognostic signs and the role of operative management in acute pancreatitis. Surg Gynecol Obstet 1974;139:69-81.

32. Gerasimenko JV, Gryshchenko O, Ferdek PE, et al. $\mathrm{Ca}^{2+}$ release-activated $\mathrm{Ca}^{2+}$ channel blockade as a potential tool in antipancreatitis therapy. Proc Natl Acad Sci USA 2013;110:13186-91. 